\title{
Influence of affordability of alcohol on educational disparities in alcohol-related mortality in Finland and Sweden: a time series analysis
}

\author{
Kimmo Herttua, ${ }^{1,2}$ Olof Östergren, ${ }^{3}$ Olle Lundberg, ${ }^{3}$ Pekka Martikainen ${ }^{2,3,4}$
}

\begin{abstract}
- Additional material is published online only. To view please visit the journal online (http://dx.doi.org/10.1136/ jech-2017-209636).

1 Department of Public Health, Centre of Maritime Health and Society, University of Southern Denmark, Esbjerg, Denmark ${ }^{2}$ Department of Social Research, Population Research Unit, University of Helsinki, Helsinki, Finland

${ }^{3}$ Centre for Health Equity Studies, Stockholm University and Karolinska Institutet, Stockholm, Sweden

${ }^{4}$ The Max Planck Institute for Demographic Research, Rostock, Germany
\end{abstract}

\section{Correspondence to} Dr Kimmo Herttua, Public Health, CMSS, University of Southern Denmark, Esbjerg 6700, Denmark; kherttua@ health.sdu.dk

Received 26 June 2017 Revised 19 September 2017 Accepted 20 September 2017 Published Online First 23 October 2017
Background Prices of alcohol and income tend to influence how much people buy and consume alcohol. Price and income may be combined into one measure, affordability of alcohol. Research on the association between affordability of alcohol and alcohol-related harm is scarce. Furthermore, no research exists on how this association varies across different subpopulations. We estimated the effects of affordability of alcohol on alcohol-related mortality according to gender and education in Finland and Sweden.

Methods Vector-autoregressive time series modelling was applied to the quarter-annual aggregations of alcohol-related deaths and affordability of alcohol in Finland in 1988-2007 and in Sweden in 1991-2008. Alcohol-related mortality was defined using information on both underlying and contributory causes of death. We calculated affordability of alcohol index using information on personal taxable income and prices of various types of alcohol.

Results Among Finnish men with secondary education, an increase of $1 \%$ in the affordability of total alcohol was associated with an increase of $0.028 \%(95 \% \mathrm{Cl}$ 0.004 to 0.053 ) in alcohol-related mortality. Similar associations were also found for affordability for various types of alcohol and for beer only in the lowest education group. We found few other significant positive associations for other subpopulations in Finland or Sweden. However, reverse associations were found among secondary-educated Swedish women.

Conclusions Overall, the associations between affordability of alcohol and alcohol-related mortality were relatively weak. Increased affordability of total alcoholic beverages was associated with higher rates of alcohol-related mortality only among Finnish men with secondary education.

\section{INTRODUCTION}

Higher prices of alcohol are related to less alcohol-attributable harm according to systematic reviews. ${ }^{12}$ As in the case of many commodities, prices and income may influence how much people buy and consume alcohol. Price and income may be combined into one measure, namely, affordability of alcohol.

Research on the association between affordability of alcohol and alcohol-related harm is scarce or non-existent. A recent study based on aggregate data from Australia found only marginal effects of affordability of alcohol on alcohol consumption. ${ }^{3}$ Similarly, the association was weak between minimum prices for alcohol and alcohol-related mortality in Finland according to a previous study. ${ }^{4}$ Furthermore, no research exists how the association between affordability of alcohol and harm varies across different subpopulations. In particular, previous literature has shown large and consistent differences in alcohol-related mortality and morbidity between educational groups. ${ }^{5-9}$ However, it remains unclear how affordability has changed in the different education groups and whether alcohol-related mortality is more sensitive to affordability changes in some education groups.

Increased affordability may have different effects on alcohol consumption and harm. On the one hand, increased affordability may increase alcohol-related harm because of increased excess consumption, that is, harmful drinking. On the other hand, increased affordability may not increase alcohol-related harm. This could be the case if alcohol consumption does not increase, or if it increased only to the extent that it remains within the limits of moderate drinking. Increased affordability may also, instead of increasing total consumption, influence people's choices so that they purchase more expensive beverages of a better quality, ${ }^{10}$ or they purchase more beverages but instead of consuming more, store them for later use. ${ }^{11}$

Possible differential effects of increased affordability can be understood according to principles of price elasticity of demand which is a measure of the responsiveness of demand for a product to change in price. Demand is said to be elastic when a small change in price producers a large change in demand. ${ }^{12}$ Price elasticity of demand tends to be smaller in countries with high income levels, and on the other hand, consumers with limited incomes tend to be more price conscious. ${ }^{13}$ Furthermore, for goods that can be stored easily, such as alcohol beverages, short-run demand may be more elastic than long-run demand. ${ }^{11}$ One can namely buy large quantities of such goods, for example, when a product goes on sale, without increasing consumption but for storing them. ${ }^{11}$

Therefore, we estimated the association between affordability of alcohol overall and different types of alcoholic beverages and alcohol-related mortality among men and women across three educational groups in Finland and Sweden using time series analysis. Analyses on Finland and Sweden provide a good opportunity to compare two neighbouring countries with many similarities, such as high average income, welfare-state system, 
alcohol monopolies that set the retail price for most drinks and register-based data of high quality, but at the same time great differences in terms of levels of alcohol consumption and alcohol-related harm, with both being higher in Finland. ${ }^{14-17}$ The data on affordability of alcohol, based on price indices for alcoholic beverages and personal taxable income, education and deaths were derived from nationwide registers. Due to the state monopolies for selling alcohol and national registers, accurate information on pricing, disposable income and alcohol-related mortality is available. Finland and Sweden represent thus ideal settings for studying the association between affordability and alcohol-related mortality.

\section{METHODS}

\section{Overview of the research design}

The present study addresses the association between affordability of alcohol overall and for different types of alcoholic beverages and alcohol-related mortality among men and women aged between 30 and 79 across three educational groups in Finland and Sweden. To do this, we created quarterly (3-month period) time series data on alcohol-related mortality using individual-level Finnish and Swedish national registers. Affordability of alcohol is a combined measure of price and income for which the information was obtained from national registers and data files. These data for the periods 1988-2007 (Finland) and 19912008 (Sweden) were analysed by means of vector-autoregressive (VAR) time series modelling.

\section{Study population}

Our mortality data for Finland were based on the individual-level Statistics Finland Labour Market data covering all residents in Finland with a linkage to death records during the period 1 January 1988 to 31 December 2007. Statistics Finland provided a nationally representative $11 \%$ sample of the whole data set, the maximum allowed by data protection regulations covering living individuals, and an additional oversample of those who died, so that in total the data covered $80 \%$ of all deaths.

We used the Swedish Work and Mortality Database, which contains linked data from several national Swedish registers. The data covered the period 1 January 1991 to 31 December 2008. We restricted the sample in this study to persons aged 30-79 years. The age span 30-79 was chosen because (1) education is stable in this age group and prior studies indicate that the majority of alcohol-related deaths occur in this age group, ${ }^{57}$ and (2) health among people younger than 30 is not very responsive to price changes in Finland. ${ }^{7}$

\section{Follow-up for alcohol-related mortality}

Causes of death were classified according to Finnish and Swedish versions of the International Statistical Classification of Diseases and Related Health Problems, 9th and 10th revision (ICD-9 and ICD-10). Alcohol-related deaths were defined as those for which there was a reference to alcohol on the death certificate as the underlying or one of the contributory causes of death. Estimating alcohol-related mortality on the basis of both the underlying and contributory causes yields more versatile and comprehensive data than the standard method based solely on the underlying cause, particularly in Finland, where death certificates record alcohol intoxication as a contributory cause more frequently and accurately than in most other countries. ${ }^{18}$ Frequent use of medico-legal autopsy is an important factor enabling the proper attribution of alcohol intoxication as a contributory cause of death. Medico-legal autopsies were carried out in over $90 \%$ of accidental or violent deaths in people aged under 65 in Finland in 1987-2003 and in Sweden in 2012 ${ }^{19}$ and in over 60\% and $54 \%$, respectively, of all deaths among those aged under 65 in Finland and Sweden in 1987-2003. ${ }^{520}$

The total pool of alcohol-related deaths used here consists of the following two main categories: (1) the underlying cause of death was an alcohol-attributable disease or fatal alcohol poisoning; and (2) the underlying cause was not alcohol related, but a contributory cause was an alcohol-attributable disease or alcohol intoxication (ICD-10 code F100). However, alcohol intoxication was not included as a contributory cause in the Swedish data, which likely results in underestimation of alcohol-related deaths compared with the Finnish data. The first group included the following alcohol-attributable diseases: alcoholic liver disease (K70), alcohol dependence syndrome (F102), alcoholic cardiomyopathy (I426), alcoholic diseases of the pancreas (K852 and K860), other mental and behavioural disorders due to alcohol (F101 and F103-F109), fatal alcohol poisoning (X45) and a few rarely occurring categories (K292, G312, G4051, G621 and G721). In the second group, the underlying cause was cardiovascular disease, accidental falls, traffic accidents, suicides or other accidents or violence in $84 \%$ of the cases.

We used alcoholic liver diseases instead of all liver diseases because there seems not to be any strong tendency to under-report alcoholic cases in Finland or Sweden: for example, in Finland in 2006, 98\% of deaths due to liver cirrhosis among men aged under 65 were classified as alcohol related on the death certificate. $^{21}$

\section{Assessment of education}

The mortality data were stratified according to gender and education. Information on education was obtained from the National Register of Completed Education and Degrees in the case of Finland and from the LISA register (Longitudinell integrationsdatabas för sjukhusförsäkrings- och arbetsmarknadsstudier) kept by the Statistics Sweden. The three educational categories were based on the highest educational qualification: basic education referring to primary or lower secondary education (International Standard Classification of Education (ISCED) codes 0-2), secondary education referring to upper secondary education (ISCED codes 3-4) and tertiary education (ISCED 5-6).

\section{Affordability of alcohol}

In affordability of alcohol, two concepts, that is, price of alcohol and personal income, are combined to one measure. The affordability of alcohol was calculated as the average income divided by the real alcohol price index. ${ }^{322}$ Income, based on the information provided by the Finnish tax administration and the Social Insurance Institution for Finland and by LISA register for Sweden, was measured as individual taxable income, comprising all forms of taxable income, including wages, capital income and taxable income transfers, and excluding certain social benefits and allowances not subject to taxation separately by sex and education. We used linear interpolation to convert the yearly income to the quarterly one.

The retail sales price indices are obtained from the national statistics. The Finnish series are compiled by the National Institute for Health and Welfare by combining alcoholic beverage price indices prepared by the Finnish retail monopoly (Alko) and the grocery shop alcoholic beverage price indices for low-alcohol-content drinks calculated by Statistics Finland using annual shares of the value of consumption as weights. The Swedish price 
Table 1 Number of alcohol-related deaths and mortality rates per 100000 person-years by education among Finnish (1988-2007) and Swedish (1991-2008) men and women aged 30-79 years

\begin{tabular}{|c|c|c|c|c|}
\hline \multirow{2}{*}{$\begin{array}{l}\text { Sex and } \\
\text { education }\end{array}$} & \multicolumn{2}{|l|}{ Finland } & \multicolumn{2}{|l|}{ Sweden } \\
\hline & Deaths & Rate & Deaths & Rate \\
\hline \multicolumn{5}{|l|}{ Men } \\
\hline Tertiary & 6100 & 73.3 & 1728 & 15.8 \\
\hline Secondary & 17006 & 127.6 & 8605 & 44.7 \\
\hline Basic & 29188 & 204.8 & 12705 & 80.2 \\
\hline All & 52294 & 144.7 & 23038 & 46.9 \\
\hline \multicolumn{5}{|l|}{ Women } \\
\hline Tertiary & 1321 & 13.0 & 632 & 5.3 \\
\hline Secondary & 3041 & 23.8 & 2228 & 11.7 \\
\hline Basic & 5928 & 37.9 & 2793 & 17.9 \\
\hline All & 10290 & 27.9 & 5653 & 11.6 \\
\hline
\end{tabular}

indices based on data from the Swedish retail monopoly (Systembolaget) are calculated similarly by Statistics Sweden. We used price indices based on current prices instead of inflation-adjusted prices. Nominal prices, as opposed to real prices, are simpler and less vulnerable to unexpected effects resulting from specific products driving changes in the retail price index. ${ }^{22}$

\section{Statistical analysis}

For the time series analyses, we converted alcohol-related deaths to quarterly death rates per 100000 person-years, separately for men and women across the three educational groups. Mortality series were not standardised for age because ageing produces trends in time series and in the analyses these trends can be removed, usually by differencing the time series. ${ }^{23}$ We used VAR modelling to analyse the association between the quarterly series of affordability of alcohol and alcohol-related mortality. VAR modelling makes it possible to describe the interaction of variables through time in a complex multivariate system. ${ }^{23}$ One of its main features is its ability to investigate causal relationships between variables. $^{24} 25$

We applied a two-variable VAR model in the study including the affordability of alcohol and alcohol-related mortality in the following two simultaneous equations:

$$
\begin{aligned}
\text { Affordability }_{t}= & \beta_{10}+\beta_{11} \text { Mortality }_{t-1}+\ldots+\beta_{1 p} \text { Mortality }_{t-p} \\
& +\alpha_{11} \text { Affordability }_{t-1}+\ldots+\alpha_{1 p} \text { Affordability }_{t-p}+\varepsilon_{1 t}
\end{aligned}
$$

Mortality $_{t}=\beta_{20}+\beta_{21}$ Mortality $_{t-1}+\ldots+\beta_{2 p}$ Mortality $_{t-p^{+}}$ $\alpha_{21}$ Affordability $_{t-1}+\ldots+\alpha_{2 p}$ Affordability $_{t-p}+\varepsilon_{2 t}$

where $\alpha$ s and $\beta$ s are unknown coefficients, $t$ is time, $\mathrm{p}$ is a number of lags and $\varepsilon_{1 \mathrm{t}}$ and $\varepsilon_{2 \mathrm{t}}$ are error terms. We show results that are based on the latter equation. We captured seasonality, when necessary, by including seasonal dummies as exogenous variables. We also ran VAR models including alcohol price index and average income separately, in addition to the combined measure of affordability, and alcohol-related mortality. We also performed a sensitivity analysis, including an indicator variable for a large reduction in Finnish excise tax on alcohol in March 2004. ${ }^{26}$ However, the estimates changed very little.

Both affordability and mortality series in VAR models need to be stationary, indicating that there is no systematic change in mean or variance over time in the series. We performed the Augmented Dickey-Fuller test in order to explore this (online supplementary eTable 1). When a series turned out to be non-stationary, we employed differencing to the series. VAR estimation requires a lag structure to be specified for the model, so we used the Schwarz Bayesian information criterion (SBIC), the Hannan-Quinn information criterion (HQIC) and the Akaike information criterion (AIC) statistics to determine the optimal number of lags to include. ${ }^{27}$ After estimating the VAR models, we used the SBIC, HQIC and AIC to determine the choice between competing models. In addition, we calculated orthogonalised impulse response functions that show the percentage change over time in mortality in response to a $1 \%$ change in the affordability of alcohol. All analyses were conducted in Stata/MP V.14.2, and we considered a two-sided $\mathrm{p}$ value of $<0.05$ significant.

\section{RESULTS \\ Descriptive results}

Table 1 shows that 52294 men and 10290 women in Finland in 1988-2007 and 23038 men and 5653 women in Sweden in 1991-2008 died from alcohol-related causes. This means that $84 \%$ and $80 \%$ of these deaths, respectively, occurred among men in Finland and Sweden. Mortality rates were approximately threefold among those with only basic education as compared with those with tertiary education for Finnish men and women and Swedish women, but around fivefold for Swedish men. Figure 1 illustrates that alcohol-related mortality rates in Finland began to increase among the less-educated men and women, in particular, in the late 1990s. In Sweden, the mortality rates seem to change much less. However, a visual inspection shows some increase among the less-educated Swedes after the beginning of the 2000s.

Affordability of total alcohol in different educational levels among men and women in Finland and in Sweden is shown in figure 2. Affordability of alcohol doubled between 1988 and 2007 among Finnish higher-educated men and women, whereas the increase was somewhat less pronounced among the low educated. A remarkable rise in the affordability of alcohol in Finland in the beginning of 2004 was due to tax cuts of alcohol following the changes in Finnish alcohol legislation. ${ }^{26}$ In Sweden, the increase in affordability from 1991 to 2008 was largest among the highest-educated men, but among women, it was similar irrespective of the educational level.

\section{Time series analysis of affordability of alcohol on alcohol-related mortality}

The association between affordability of alcohol and alcohol-related mortality by education among men and women in Finland and Sweden was assessed using VAR models. To present the results of these models in an informative and easily understandable way, we show the effects of a $1 \%$ change in the affordability of total alcohol on alcohol-related mortality over a period of 10 annual quarters (tables 2 and 3, figures 3 and 4 and online supplementary etables 2-5). These effects are obtained from an orthogonalised impulse response functions of the VAR models. Among Finnish men with secondary education, an increase (decrease) of $1 \%$ in the affordability of total alcohol was associated with an increase (decrease) of $0.028 \%$ (95\% CI $0.004 \%$ to $0.053 \%$ ) in alcohol-related deaths per 100000 person-years. Similar associations among them were found for distilled spirits, wine, strong beer and medium beer, the estimates being $0.026 \%(95 \%$ CI $0.001 \%$ to $0.051 \%$ ), $0.025 \%$ (95\% CI $0.001 \%$ to $0.049 \%$ ), $0.028 \%$ (95\% CI $0.003 \%$ to $0.053 \%$ ) and $0.026 \%(95 \% \mathrm{CI}$ $0.001 \%$ to $0.051 \%)$, respectively. 

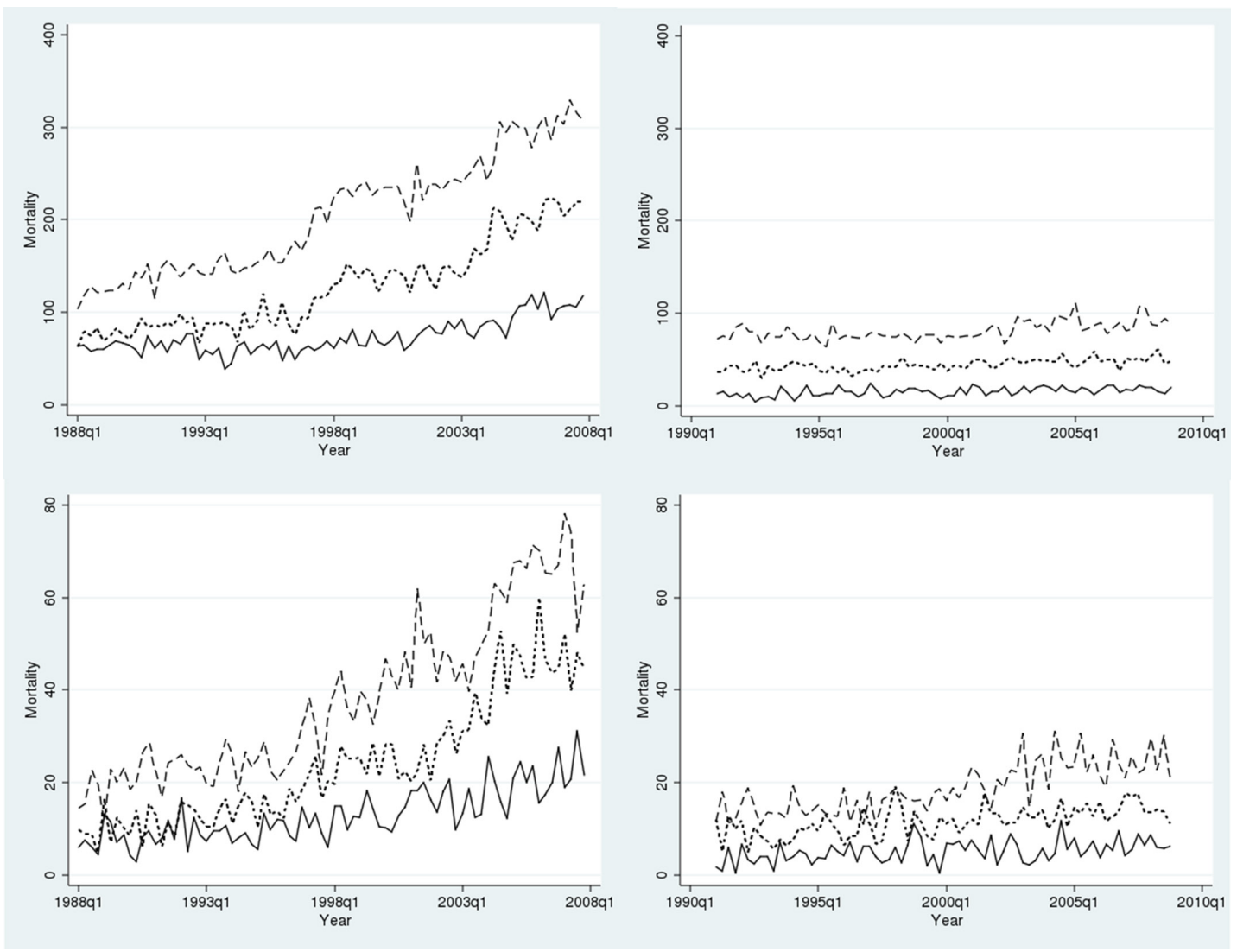

Figure 1 Alcohol-related mortality by education among Finnish and Swedish men (upper panels), and Finnish and Swedish women (lower panels) in 1988-2007 (Finland) and in 1991-2008 (Sweden). Tertiary (solid line), secondary (dotted line) and basic (dashed line) education. q1, first quarter.

A statistically significant association was also found among Finnish men with basic education for medium beer, the estimates being $0.019 \%$ (95\% CI $0.001 \%$ to $0.037 \%$ ). In contrast, an increase (decrease) of $1 \%$ in affordability of total alcohol was associated with a decrease (increase) of $0.041 \%$ (95\% CI $0.006 \%$ to $0.076 \%$ ) in total alcohol-related mortality among Swedish women with secondary education. A similar association was found for distilled spirits, wine and beer and alcohol-related mortality among Swedish women with secondary education and for beer and alcohol-related mortality among Swedish women combined. Otherwise, we found no association for all men or women combined.

VAR models that included the alcohol price index and average income separately, instead of the combined measure of affordability of alcohol, and alcohol-related mortality showed that impact of price is greater than that of income on alcohol-related mortality (online supplementary etable 6 and 7). However, no statistically significant association was found.

\section{DISCUSSION}

In this large-scale, population-based time series analysis from Finland and Sweden, we observed that affordability of alcohol and alcohol-related mortality was associated only in certain population subgroups and only for certain types of alcoholic beverages. Finnish men with secondary education had increased mortality rates in response to the increased affordability of total alcohol and different types of alcoholic beverages. We also found similar effects among Finnish men with basic education for medium beer. In contrast, a decrease in alcohol-related mortality among Swedish women with secondary education was associated with an increase in affordability of total alcohol and different types of alcoholic beverages. The increased affordability of total alcohol was not related to higher rates of alcohol-related mortality among all men or women in Finland or in Sweden.

To our best knowledge, this is the first study on the association of affordability of alcohol and alcohol-related mortality. However, our results are in accordance with findings from a recent study from Australia, which found only a marginal association between affordability of alcohol and alcohol consumption. ${ }^{3}$ In the same study, beverage-specific analyses on the effects of prices instead of affordability show that a $1 \%$ increase in the price of spirits led to a decrease in consumption of spirits by $0.03 \%$ in the second year. However, this effect disappeared in the third year. ${ }^{3}$ A recent study from Finland also showed a relatively marginal association between minimum prices of alcohol and alcohol-related mortality in Finland. ${ }^{4}$ Among Finnish men with a basic education, an increase of $1 \%$ in the minimum price of alcohol was associated with a decrease of $0.03 \%$ in alcohol-related mortality, whereas among the most highly educated, there were no associations between the minimum prices of any beverages and mortality. ${ }^{4}$ The finding among the basic educated is consistent with the findings of this study on affordability of medium beer and alcohol-related mortality. Overall, recent evidence from Finland seems to indicate that although price changes seemed to affect the lowest socioeconomic groups most in terms of harm-particularly in absolute terms and among men-these effects were sometimes weak and not consistent by gender and across different measures of harm. ${ }^{28}$ Moreover, a recent study from Denmark found that, in spite of increases in affordability of alcohol in 2006-2011, the overall alcoholic liver disease incidence decreased along with per capita consumption. ${ }^{29}$ The relationship between incidence of alcoholic liver 

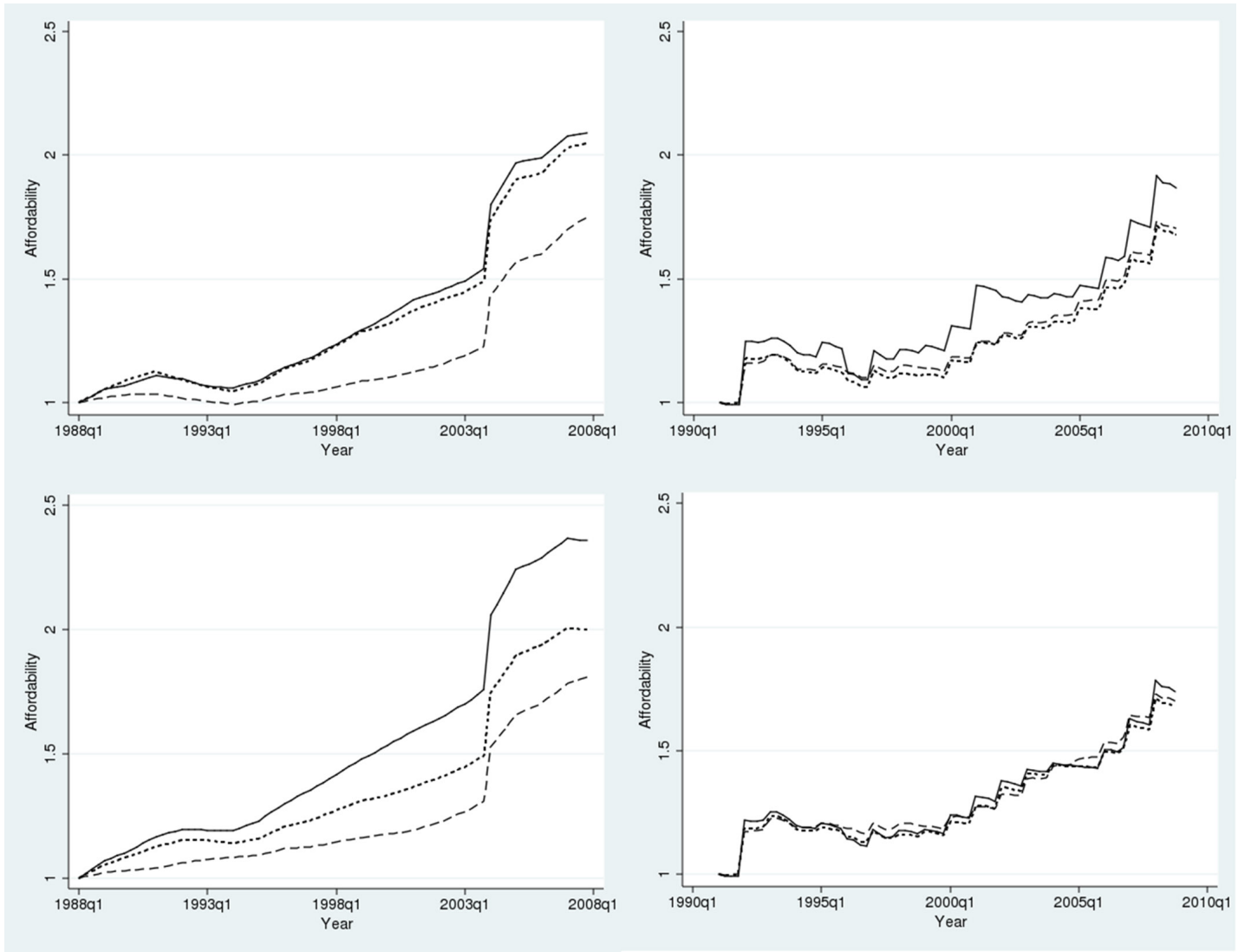

Figure 2 Affordability of alcohol by education among Finnish and Swedish men (upper panels) and Finnish and Swedish women (lower panels) in 1988-2007 (Finland) and in 1991-2008 (Sweden). Tertiary (solid line), secondary (dotted line) and basic (dashed line) education. q1, first quarter.

disease and affordability of alcohol was not formally tested in this study.

Although the trends in affordability of alcohol and the levels and trends in mortality differ largely between Finland and Sweden, the overall association between affordability of alcohol and alcohol-related mortality was weak in both countries. This relatively

Table 2 Orthogonalised impulse response functions from vectorautoregressive models of the estimated short-term effect of affordability of alcohol on alcohol-related mortality according to education among men, Finland in 1988-2007 and Sweden in 1991-2008

\begin{tabular}{|c|c|c|c|c|c|}
\hline \multicolumn{2}{|l|}{$\begin{array}{l}\text { Country and } \\
\text { education }\end{array}$} & \multicolumn{2}{|c|}{ Seasonally unadjusted VAR } & \multicolumn{2}{|c|}{ Seasonally adjusted VAR } \\
\hline & Lags & OIRF & $95 \% \mathrm{Cl}$ & OIRF & $95 \% \mathrm{Cl}$ \\
\hline \multicolumn{6}{|l|}{ Finland } \\
\hline Tertiary & 1 & 0.004 & -0.028 to 0.036 & -0.002 & -0.034 to 0.029 \\
\hline Secondary & 1 & 0.028 & 0.004 to 0.053 & 0.023 & 0.000 to 0.046 \\
\hline Basic & 1 & 0.008 & -0.009 to 0.025 & 0.000 & -0.015 to 0.016 \\
\hline All & 1 & 0.016 & 0.001 to 0.030 & 0.007 & -0.005 to 0.019 \\
\hline \multicolumn{6}{|l|}{ Sweden } \\
\hline Tertiary & 1 & 0.002 & -0.096 to 0.101 & 0.011 & -0.068 to 0.090 \\
\hline Secondary & 3 & -0.020 & -0.041 to 0.002 & -0.020 & -0.041 to 0.002 \\
\hline Basic & 1 & -0.022 & -0.048 to 0.004 & 0.006 & -0.016 to 0.027 \\
\hline All & 1 & -0.003 & -0.022 to 0.017 & 0.012 & -0.003 to 0.028 \\
\hline
\end{tabular}

Model estimates in bold indicate models with a better fit according to Schwarz Bayesian, Hannan-Quinn and Akaike information criteria.

OIRF, orthogonalised impulse response function; VAR, vector autoregressive. weak overall association between affordability of alcohol and alcohol-related mortality may be accounted for by the fact that both Finland and Sweden are high-income countries. Prices of alcohol may therefore have not been an obstacle for drinking, not even among the less educated, because price elasticities tend to be smaller in high-income countries. ${ }^{13}$ The observed association

Table 3 Orthogonalised impulse response functions from vectorautoregressive models of the estimated short-term effect of affordability of alcohol on alcohol-related mortality according to education among women, Finland in 1988-2007 and Sweden in 1991-2008

\begin{tabular}{|c|c|c|c|c|c|}
\hline \multicolumn{2}{|l|}{$\begin{array}{l}\text { Country and } \\
\text { education }\end{array}$} & \multicolumn{2}{|c|}{ Seasonally unadjusted VAR } & \multicolumn{2}{|c|}{ Seasonally adjusted VAR } \\
\hline & Lags & OIRF & $95 \% \mathrm{Cl}$ & OIRF & $95 \% \mathrm{Cl}$ \\
\hline \multicolumn{6}{|l|}{ Finland } \\
\hline Tertiary & 1 & 0.015 & -0.064 to 0.094 & 0.019 & -0.080 to 0.084 \\
\hline Secondary & 1 & 0.039 & -0.019 to 0.098 & 0.027 & -0.031 to 0.085 \\
\hline Basic & 1 & 0.026 & -0.017 to 0.070 & 0.023 & -0.019 to 0.066 \\
\hline All & 1 & 0.028 & -0.007 to 0.063 & 0.023 & -0.012 to 0.057 \\
\hline \multicolumn{6}{|l|}{ Sweden } \\
\hline Tertiary & 1 & -0.037 & -0.238 to 0.163 & 0.025 & -0.011 to 0.160 \\
\hline Secondary & 1 & -0.041 & -0.076 to -0.006 & -0.019 & -0.044 to 0.007 \\
\hline Basic & 3 & -0.021 & -0.050 to 0.008 & -0.008 & -0.038 to 0.021 \\
\hline All & 3 & -0.026 & -0.052 to 0.000 & -0.019 & -0.044 to 0.007 \\
\hline
\end{tabular}

Model estimates in bold indicate models with a better fit according to Schwarz Bayesian, Hannan-Quinn and Akaike information criteria.

OIRF, orthogonalised impulse response function; VAR, vector autoregressive. 

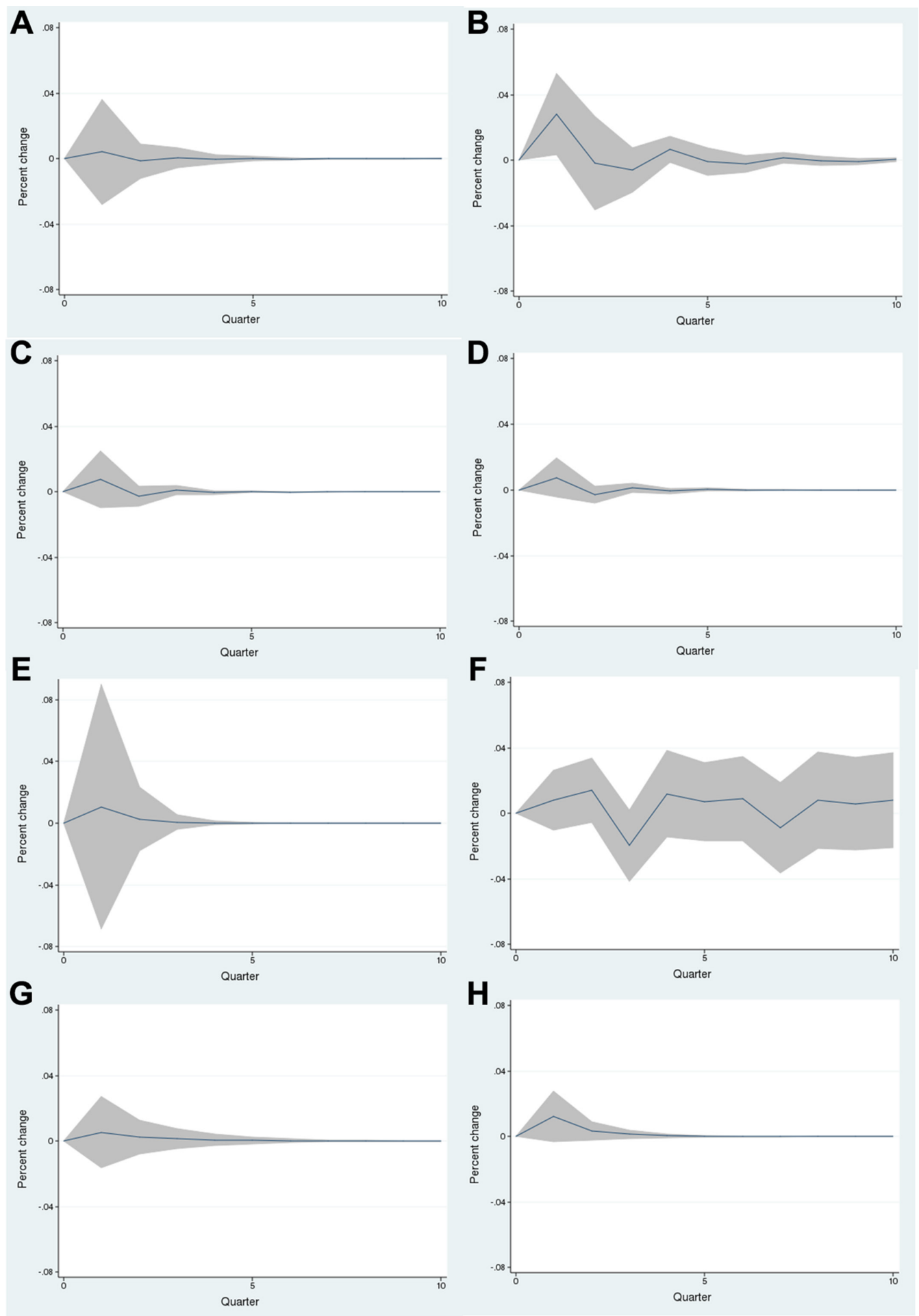

Figure 3 Impact of affordability of alcohol total on alcohol-related mortality by education among Finnish and Swedish men in 1988-2007 (Finland) and in 1991-2008 (Sweden). The graphs for the subgroups from top left to bottom right are as follows: Finnish (A) tertiary education, (B) secondary education, (C) basic education, (D) all; Swedish (E) tertiary education, (F) secondary education, (G) basic education, (H) all. Orthogonalised impulse response functions based on vector-autoregressive models.

between affordability and mortality among Finnish men with secondary education is in agreement with results from a study on effects of alcohol price changes on alcohol-related mortality in Finland, according to which the greatest proportional increase in alcohol-related mortality occurred among men with a secondary education. ${ }^{7}$ The finding that alcohol affordability was associated with alcohol-related mortality among Finnish men with basic education for medium beer may be accounted for their beverage preferences and drinking pattern. According to a survey, men with a basic education consumed $61 \%$ of their total ethanol intake as beer. ${ }^{30}$ Moreover, prevalence for harmful drinking is higher among them compared with other educational groups. ${ }^{31}$

A particular strength of our study is that we were able to use datasets of high quality from two different countries. For Finland, we 

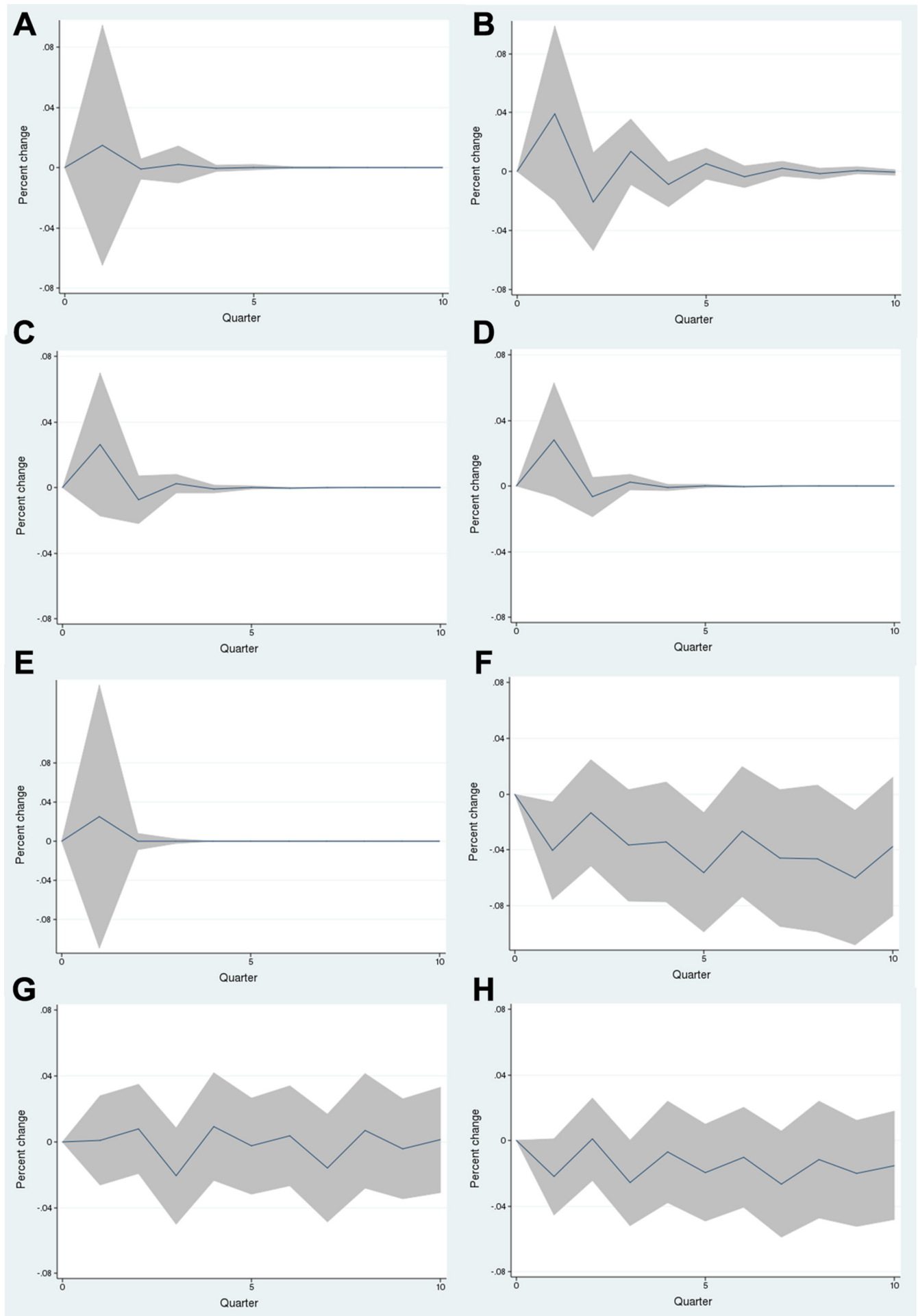

Figure 4 Impact of affordability of alcohol total on alcohol-related mortality by education among Finnish and Swedish women in 1988-2007 (Finland) and in 1991-2008 (Sweden). The graphs for the subgroups from top left to bottom right are as follows: Finnish (A) tertiary education, (B) secondary education, (C) basic education, (D) all; Swedish (E) tertiary education, (F) secondary education, (G) basic education, (H) all. Orthogonalised impulse response functions based on vector-autoregressive models.

used the large population-based sample, with an $80 \%$ oversample of deaths. Yielding information on both underlying and contributory causes of death, based often on autopsy, these mortality data are likely to capture the full range of alcohol-related deaths. The Finnish death register, which we used, is highly ranked in international comparisons with respect to reliability and accuracy. ${ }^{32}$ For Sweden, we used a total population register with near-universal coverage. Therefore, bias because of coding artefacts is an unlikely explanation of our results. Moreover, the ability to compare Finland and Sweden is a strength; although neighbouring countries with many similarities, for example, in terms of welfare-state system and high average income, they have also significant differences in levels and trends of alcohol-related mortality, trends of affordability and in alcohol policy. There has been, for example, a more rapid increase in alcohol-related mortality in Finland during the recent decades. 
There are, nevertheless, limitations to this study. First, our analysis was stratified by sex and education, but stratified data on alcohol consumption, the most evident direct causal factor for alcohol-related mortality, were not available. However, previous evidence from Finland suggests that heavy drinkers are the most sensitive to price decreases in terms of mortality and morbidity. ${ }^{73-35}$ This is likely also in the case of affordability for the time-scale we used. Second, like all observational epidemiological studies, this one was subject to residual confounding by unobserved risk factors. Some factors, such as unemployment rate, could have contributed to changes in alcohol-related mortality. For these factors to bias the estimated relation between affordability of alcohol and mortality, their temporal variation should coincide with those of affordability and mortality. Including confounding factors is therefore less relevant in time series models compared with regression models. Third, although we have estimated the optimal number of lags to include in the analyses, it is not possible to capture the full impact of affordability of alcohol on mortality mainly because of a long latency period for alcohol-related diseases. It has been suggested, for instance, that the full effect of alcohol consumption on liver cirrhosis mortality is distributed over as many as 40 years. ${ }^{36}$ However, an earlier study on the health effects of drastic policy changes of alcohol in Finland in 2004 on alcohol-related mortality showed that the effect of a sudden price change may be instant, even in terms of liver cirrhosis. ${ }^{7}$ In any case, results of this study need to be interpreted as short-term rather than as long-term effects of affordability of alcohol. Finally, because of exclusion of persons aged less than 30 years in this study, future research should address the association between affordability of alcohol and alcohol-related harm at younger ages.

In conclusion, we investigated the association between affordability of alcohol and alcohol-related mortality stratified by sex and education in Finland and Sweden using time series analysis. Although for affordability of total alcohol, this association in general was weak, a stronger association was found among Finnish men with secondary education. We also found a similar

\section{What is already known on this subject}

- Although the pricing of alcohol is regarded as an effective tool in reducing alcohol-related harm, research on the association between affordability of alcohol and alcoholrelated harm is scarce or non-existent.

- There is evidence of marginal association between an increase of affordability of alcohol and an increase in alcohol consumption in Australia, but no analyses were done across different population groups. Similarly, a weak association has been found between minimum prices for alcohol and alcoholrelated mortality in Finland.

\section{What this study adds}

- To our knowledge, this is the first study investigating the effects of affordability of alcohol on alcohol-related mortality across different subpopulations of different countries.

- Our data suggest that associations between affordability and alcohol-related mortality are generally weak, but a stronger association was found among Finnish men with secondary education. effect among Finnish men with basic education for affordability of medium beer. Overall, this study does not give impregnable support to the idea that intervening on the affordability of alcohol is an effective measure to tackle alcohol-related health problems in all contexts and for all social strata. The reasons for this are unclear, but the relative desirability and affordability of alcohol in relation to other consumption items is likely to play a role. In addition to the price instrument preventive actions among high-risk groups and measures specifically targeted to problem drinkers instead of the whole population also need to be considered in order to reduce alcohol-related harm and inequalities in alcohol-related mortality.

Acknowledgements We thank Statistics Finland for granting access to the data set (permission TK-53-508-09).

Contributors $\mathrm{KH}$ and PM designed the study. $\mathrm{KH}$ performed data analysis and wrote the manuscript. PM, $\mathrm{OO}$ and $\mathrm{OL}$ contributed to writing of the manuscript. All authors had full access to all of the data (including statistical reports and tables) in the study and can take responsibility for the integrity of the data and the accuracy of the data analysis. $\mathrm{KH}$ is the guarantor.

Funding PM is supported by the Academy of Finland. This study is part of the DEMETRIQ project. The research leading to these results has received funding from the Commission of the European Communities Seventh Framework Programme under grant agreement number 278511. The study does not necessarily reflect the Commission's views and in no way anticipates the Commission's future policy in this area. This research was also conducted as part of the Nordic project, Contingent Life Courses (C-LIFE), funded by Nordforsk, the Nordic Programme on Health and Welfare (grant number 75970). The funders had no role in the study design, the data collection and analysis, the decision to publish or the preparation of the manuscript.

Competing interests All authors have completed the ICMJE uniform disclosure form at www.icmje.org/coi disclosure.pdf (available on request from the corresponding author) and declare: no support from any organisation for the submitted work; no financial relationships with any organisations that might have an interest in the submitted work in the previous three years; no other relationships or activities that could appear to have influenced the submitted work.

Ethics approval Statistics Finland gave ethical approval for this study.

Provenance and peer review Not commissioned; externally peer reviewed.

Data sharing statement No additional data are available.

(C) Article author(s) (or their employer(s) unless otherwise stated in the text of the article) 2017. All rights reserved. No commercial use is permitted unless otherwise expressly granted.

\section{REFERENCES}

1 Wagenaar AC, Tobler AL, Komro KA. Effects of alcohol tax and price policies on morbidity and mortality: a systematic review. Am J Public Health 2010;100:2270-8.

2 Anderson P, Chisholm D, Fuhr DC. Effectiveness and cost-effectiveness of policies and programmes to reduce the harm caused by alcohol. Lancet 2009;373:2234-46.

3 Jiang $\mathrm{H}$, Livingston $\mathrm{M}$. The dynamic effects of changes in prices and affordability on alcohol consumption: an impulse response analysis. Alcohol Alcohol 2015;50:631-8.

4 Herttua K, Mäkelä P, Martikainen P. Minimum prices for alcohol and educational disparities in alcohol-related mortality. Epidemiology 2015;26:337-43.

5 Herttua K, Mäkelä P, Martikainen P. Differential trends in alcohol-related mortality: a register-based follow-up study in Finland in 1987-2003. Alcohol Alcohol 2007;42:456-64

6 Herttua K, Mäkelä P, Martikainen P. Educational inequalities in hospitalisation attributable to alcohol: a population-based cohort study. Addiction 2015;110:1092-100.

7 Herttua K, Mäkelä P, Martikainen P. Changes in alcohol-related mortality and its socioeconomic differences after a large reduction in alcohol prices: a natural experiment based on register data. Am J Epidemiol 2008;168:1110-8.

8 Probst C, Roerecke M, Behrendt S, et al. Socioeconomic differences in alcoholattributable mortality compared with all-cause mortality: a systematic review and meta-analysis. Int J Epidemiol 2014;43:1314-27.

9 Menvielle G, Kunst AE, Stirbu I, et al. Socioeconomic inequalities in alcohol related cancer mortality among men: to what extent do they differ between Western European populations? Int J Cancer 2007;121:649-55.

10 Gruenewald PJ, Ponicki WR, Holder HD, et al. Alcohol prices, beverage quality, and the demand for alcohol: quality substitutions and price elasticities. Alcohol Clin Exp Res 2006;30:96-105 
11 Perloff JM. Microeconomics. Theory and applications with calculus. international edition. Boston, USA: Pearson Education Inc, 2008: p 38.

12 Pindyck RS, Rubinfeld D. Microeconomics. 8th. ed. UK: Pearson Education Limited, 2015:p33.

13 Hill CWL. International business: competing in the global marketplace. 10th. ed. New York, USA: McGraw-Hill Education, 2015:p553.

14 GBD Risk Factors Collaborators. Global, regional, and national comparative risk assessment of 79 behavioural, environmental and occupational, and metabolic risks or clusters of risks, 1990-2015: a systematic analysis for the Global Burden of Disease Study 2015. Lancet 2015;2016:1659-724.

15 GBD 2015 DALYs and HALE Collaborators. Global, regional, and national disabilityadjusted life-years (DALYs) for 315 diseases and injuries and healthy life expectancy (HALE), 1990-2015: a systematic analysis for the Global Burden of Disease Study 2015. Lancet 2016;388:1603-58.

16 GBD 2015 Disease and Injury Incidence and Prevalence Collaborators. Global, regional, and national incidence, prevalence, and years lived with disability for 310 diseases and injuries, 1990-2015: a systematic analysis for the Global Burden of Disease Study 2015. Lancet 2016;388:1545-602.

17 GBD 2015 Mortality and Causes of Death Collaborators. Global, regional, and national life expectancy, all-cause mortality, and cause-specific mortality for 249 causes of death, 1980-2015: a systematic analysis for the Global Burden of Disease Study 2015. Lancet 2016;388:1459-544.

18 Lahti RA, Penttilä A. The validity of death certificates: routine validation of death certification and its effects on mortality statistics. Forensic Sci Int 2001;115:15-32.

19 Statistics Finland. Causes of death 2007. Helsinki: Official Statistics offinland, 2009.

20 Socialstyrelsen Causes of Death2012. http://www.socialstyrelsen.se/register/ dodsorsaksregistret Accessed 13 Feb 2017

21 Statistics Finland. causes of death 2006. Helsinki: Official Statistics of Finland, 2007.

22 Seabrook R. A new measure of alcohol affordability for the UK. Alcohol Alcohol 2010;45:581-5.

23 Brandt PT, Williams JT. Multiple time series models. Thousand Oaks, CA: Sage Publications, 2007

24 Rosmalen JG, Wenting AM, Roest AM, et al. Revealing causal heterogeneity using time series analysis of ambulatory assessments: application to the association between depression and physical activity after myocardial infarction. Psychosom Med 2012;74:377-86.

25 Lütkepohl H. New Introduction to Multiple Time Series Analysis. Berlin, Germany: Springer Verlag, 2006.

26 Herttua K, Martikainen P, Vahtera J, et al. Living alone and alcohol-related mortality: a population-based cohort study from Finland. PLoS Med 2011:8:e1001094.

27 Ivanov V, Kilian L. A practitioner's guide to lag-order selection for vector autoregressions, discussion papers 2685. London, UK: Centre for Economic Policy Research, 2001.

28 Mäkelä P, Herttua K, Martikainen P. The socioeconomic differences in alcohol-related harm and the effects of alcohol prices on them: a summary of evidence from Finland. Alcohol Alcohol 2015;50:661-9.

29 Deleuran T, Vilstrup H, Becker U, et al. Epidemiology of alcoholic liver disease in Denmark 2006-2011: a population-based study. Alcohol Alcohol 2015;50:352-7.

30 Metso L, Mustonen H, Makela P, et al. Finnishdrinking habits in 2000 [in Finnish]. Helsinki, Finland: National Research and Development Centre for Welfare and Health, 2002.

31 Makela P, Mustonen H, Tigerstedt H. (Eds). Finnish drinking habits in 1968<2008 [in Finnish]. Helsinki, Finland: National Institute for Health and Welfare, 2010

32 Mathers CD, Fat DM, Inoue $M$, et al. Counting the dead and what they died from: an assessment of the global status of cause of death data. Bull World Health Organ 2005:83:171-7.

33 Herttua K, Mäkelä P, Martikainen P. An evaluation of the impact of a large reduction in alcohol prices on alcohol-related and all-cause mortality: time series analysis of a population-based natural experiment. Int J Epidemiol 2011:40:441-54.

34 Herttua K, Mäkelä P, Martikainen P. The effects of a large reduction in alcohol prices on hospitalizations related to alcohol: a population-based natural experiment. Addiction 2011;106:759-67.

35 Herttua K, Mäkelä P, Martikainen $\mathrm{P}$, et al. The impact of a large reduction in the price of alcohol on area differences in interpersonal violence: a natural experiment based on aggregate data. J Epidemio/ Community Health 2008;62:995-1001.

36 Norström T. The impact of per capita consumption on Swedish cirrhosis mortality. Br J Addict 1987;82:67-75. 